\title{
Modeling the Impacts of Driver Income Distributions on Online Ride-Hailing Services
}

\author{
Yuru Wu $\mathbb{D}^{1},{ }^{1}$ Weifeng Li $\mathbb{D}^{1},{ }^{1}$ Qing Yu $\mathbb{D}^{1},{ }^{1}$ and Jinyu Chen ${ }^{2}{ }^{2}$ \\ ${ }^{1}$ Key Laboratory of Road and Traffic Engineering of the Ministry of Education, Tongji University, Shanghai 201804, China \\ ${ }^{2}$ Center for Spatial Information Science, The University of Tokyo, 5-1-5 Kashiwanoha, Kashiwa, Chiba 277-8563, Japan \\ Correspondence should be addressed to Weifeng Li; liweifeng@tongji.edu.cn
}

Received 6 July 2021; Accepted 2 September 2021; Published 20 September 2021

Academic Editor: A. M. Bastos Pereira

Copyright (c) 2021 Yuru Wu et al. This is an open access article distributed under the Creative Commons Attribution License, which permits unrestricted use, distribution, and reproduction in any medium, provided the original work is properly cited.

The online ride-hailing taxi brings new vitality into the traditional taxi market, as well as new issues and challenges. The pricing and profit distribution of online ride-hailing services is one of the major concerns. This study focuses on the pricing and income distribution in the online ride-hailing system. Queuing system model and birth and death process theory are introduced to describe the driver's flow process in the network. The social welfare maximization model and the platform profit maximization model are constructed based on the dynamic pricing mechanism, from the government's and platform's standpoint, respectively. Through numerical experiments, this paper analyzes the income distribution of drivers under different settings and the influence of different factors (average travel time, psychologically expected price of drivers and passengers, and probability of driver leaving the system) on the proportion of income distribution. The results show that the drivers' income distribution proportion is higher in the pursuit of social welfare maximization than that in the pursuit of platform profit maximization, and in different benefit pursuit models, various factors have a certain influence on the driver's income distribution proportion. The proposed method and conclusion in this study can be considered as references for online ride-hailing market supervision and policy-making.

\section{Introduction}

Ride-hailing, referring to the activity of calling a vehicle or driver to go to a destination, rises in many metropolitan areas and takes a considerable part of mobility services [1]. The online ride-hailing platform is a subversion of the traditional travel industry in the context of the Internet; it transmits the traditional service mode from the driver side to the passenger side by the combination of online information and offline experience $[2,3]$. Customers are matched efficiently by an online platform with affiliated drivers nearby by requesting rides via a mobile application [4]. Such ondemand services greatly reduce search frictions in the ridehailing market, bring passengers and drivers together at very low transaction costs, and meanwhile effectively reduce the vacancy rates of operating vehicles by matching demand and supply [5-7].

However, compared with the traditional taxi industry, the pricing and the income distribution of online ride- hailing lack transparency and consistency. The unreasonable and opaque income distribution harms the interests of drivers. On October 31, 2018, online taxi drivers in Chicago, the United States, delivered a rally and petition on the budget meeting held by the city government. They asked for more driver's share of the platform revenue. According to the survey conducted in August 2019 in the United States, the fare receipt information and more than 14756 real online taxi order service data showed that Uber averagely retains about $35 \%$ of the revenue per ride, while Lyft takes about $38 \%$. However, in the regulatory documents submitted to the government, Uber reported that its platform share of fares was only close to $20 \%$, while Lyft did not disclose its proportion publicly. What causes these situations is that the platforms can set the pricing and distribution mechanism by themselves, and under the goal of maximizing benefits, they can dynamically adjust the service price (some platforms even use dynamic pricing models for the same route, which may vary according to the demand and supply for rides) and 
the drivers' distribution proportion, which will harm the interests of drivers and passengers.

In the new traffic model, the system layout design is very important, which will affect the operational efficiency of the whole system and public acceptance [8]. Therefore, it is necessary to study the price mechanism of online ridehailing and the way of labor (drivers) drawing. However, most of the existing related research studies focus on the maximization of platform interests and seldom on the interests of drivers and study the pricing and distribution mechanisms. Therefore, from the perspective of government management, this paper constructs a social welfare maximization model to explore the relationship between social welfare and the way of interest distribution, to provide decision support for the regulation of the online ride-hailing market.

In this paper, the income distribution of drivers in the online ride-hailing market is discussed based on the dynamic pricing mechanism from the perspectives of social interest maximization and platform profit maximization. The contributions of this paper are as follows: (1) Queuing system model and birth and death process theory are introduced to describe the driver's flow process in the network. (2) The social welfare maximization model and the platform benefit maximization model are constructed, from the government's and platform's standpoint, respectively, based on the psychological expected price curve of passengers and drivers. (3) Numerical experiments are performed to analyze the driver's income distribution under the conditions of different settings. Suggestions on the government's regulation and guidance are put forward based on the experiment results.

The remainder of this paper is organized as follows. Section 2 presents a review of the related literature. Section 3 mainly focuses on the problem description and modeling. In Section 4, two numerical experiments are performed to analyze the driver's income distribution. Results and associated discussion are then carried out. Finally, conclusions and future directions are given in the last section.

\section{Literature Review}

The current discussion and studies on online ride-hailing are extensive. In terms of the price mechanism of online ridehailing, Zha et al. [9] focused on the impact of dynamic pricing on online ride-hailing industry by structurally combining driver's work schedule selection. The numerical results show that the platform enjoyed higher revenue on the basis of dynamic pricing, while customers may be exploited in the peak period of taxi service. Fellows [10] uses cost advantage analysis technology to prove that reasonable platform pricing can bring about net income for society. However, Chang [11] thinks that the pricing structure of the platform will affect the output efficiency and social welfare and points out that it disturbs the market price rule. Xia et al. [12] believe that the dynamic pricing strategy can alleviate the pressure of the platform in the period of tight transport capacity by increasing the transport capacity of part-time drivers and reducing the number of price-sensitive user orders, so as to improve user satisfaction. Banergee et al. [13] use queuing theory model to simulate the flow of drivers and passengers in online ride-hailing market for the first time and study the volume and revenue of online ride-hailing platform by static pricing and dynamic pricing. In addition, a series of literature studies [14-16] show the pricing strategy of online ride-hailing platform to explore how pricing can balance the relationship between passengers, drivers, and the platform, to maximize the benefits.

In terms of the profit distribution and drivers' income, $\mathrm{Xu}$ et al. [17] investigated the $\mathrm{C} 2 \mathrm{C}$ business model of online ride-hailing platform and concluded that, based on the technical characteristics of the online ride-hailing industry, the platforms are in an absolutely dominant position in income distribution and they can take advantage of the lower labor price of part-time drivers to force the contract drivers to reduce the labor price, thus causing a certain degree of labor market failure. Liu and Cai [18] analyzed the income distribution mode of platforms, employed drivers in China's online ride-hailing industry by using political economy theory, and demonstrated that the improvement of the exploitation rate with drivers and passengers comes from the monopoly of the platform and the profit-making nature of capital. Xie [19] made an in-depth analysis of the demand and behavior characteristics of drivers and passengers, focusing on the dynamic investigation of the income distribution proportion of drivers. The research shows that the optimal mileage price of online ride-hailing increases with the increase of tour taxi fare and decreases with the increase of "rebate." Through the analysis of the taxi platform, driver, and passenger involved in the process of online ride-hailing, Danlei [20] determined the distance, customer preference, and other influencing factors and analyzed how to maximize the interests of the three parties.

In terms of government regulation, previous studies mainly focused on the interpretation of the government's new policy on online ride-hailing and the research on the access regulation of drivers and platform based on the protection of passengers' interests. Xiang [21] believes that online ride-hailing is the product of market self-regulation, and its generation has objective market value and development prospects. Fang's study [22], based on the Bertrand model and Cournot model, constructs a repeated game with uncertain ending time and analyzes the competitive strategy of two stages of online ride-hailing market development, so as to get the path of promoting the development of online ride-hailing market. Recently, more and more attention has been paid to the supervision of pricing and distribution mechanisms. Lai [23] proposed that transparent pricing rules for online ride-hailing should be the basic consensus of the industry. Xia and Lin [24, 25] believe that government should strengthen the supervision of the pricing and distribution of online ride-hailing platforms. Li [26] proposes two problems in the government's regulation of online ridehailing market: first, the government's regulatory concept lacks active guidance and cannot actively discover new problems and carry out advance management; second, the local government mechanically implements the central level regulatory measures for the online ride-hailing market, falling into the path dependence of regulatory means. 
Previous studies explained and evaluated the proportion charged by platforms and paid less attention to the relationship between labor supply and the income distribution proportion. In addition, in terms of the income distribution, most of the studies paid attention to the interests of the platform and less attention to the rights and interests of drivers. Therefore, this paper clearly puts forward the proportion of income distribution of drivers and focuses on the rights and interests of drivers from the perspective of the government. And more importantly, this paper takes social welfare into consideration and forms a contrast with platform interests to put forward suggestions for better development of online ride-hailing.

\section{Problem Description and Modeling}

3.1. Basic Assumptions and Queuing Model. In this paper, it is assumed that the online ride-hailing market is composed of three parts: platform, driver, and passenger. The online ride-hailing system is abstracted as the queuing system shown in Figure 1. Unlike the general queuing system, since the demand of online ride-hailing market exceeds the supply, passengers in the online ride-hailing system are regarded as the service desks and drivers are regarded as the arriving customers [27]. Assuming that the new driver joins the queue of available drivers at a rate $\Lambda_{e}$, when the driver matches his own passengers, the average travel time of the passengers is $t$. After arriving at the destination, the passengers will leave the system, and the driver will either leave the system or return to the original queuing system. The probability of the driver leaving the system is set to be $q_{\text {leave }}>0$.

Assuming that the price of each ride is $p$ (yuan $/ \mathrm{km}$ ) and the driver's income distribution proportion is $A$, the driver's income of each ride is $A p$, and the income of the platform is supposed to be $(1-A) p$.

Considering the platform, passengers are usually sensitive to the price of the ride, if the price is too high, pricesensitive customers will give up the ride. And the drivers are sensitive to the average amount of money earned over a longer period of time (a few hours or a day). If the profit is too low, the driver will give up. Therefore, this paper introduces the change curve of the expected price of passengers and drivers. For passengers, assuming that the adjusted price of passengers is independent of the distribution $F_{V}$ and $V$ is the expected price of each passenger, the distribution function of $V$ is denoted as $F_{V}(V)$. If $p \leq V$, passengers are willing to ride; otherwise, they will give up. Similarly, suppose that the driver's adjusted price is independent of the distribution $F_{C}$ and $C$ is the expected revenue of each driver. The distribution function of $C$ is denoted as $F_{C}(C)$. If the actual average hourly income $A p / t \geq C$, the driver will choose to accept the order; otherwise, he will give up.

Therefore, if the initial passenger arrival rate is $\mu_{0}$, then the actual passenger arrival rate $\mu$ is

$$
\mu=\mu_{0} \overline{F_{V}}(p)=\left(1-F_{V}(p)\right) \mu_{0} .
$$

For drivers, when the order ends, the driver will leave the system with probability $q_{\text {leave }}$; meanwhile, the psychological price of drivers is independent of the distribution $F_{C}$, which affects the choice of drivers to receive orders (enter the system). Therefore, when the queue of drivers in Figure 1 is stable, the following equation relationship can be established for the probability of new drivers arriving at the system $\left(\Lambda_{e}\right)$ :

$$
\Lambda_{e}=\lambda q_{\text {leave }}=\lambda_{0} F_{C}\left(\frac{\eta}{f+t}\right)
$$

In equation (2), $f$ is the expected free time per ride, $\eta$ is the expected revenue per ride, and $\lambda_{0}$ is the initial driver arrival rate.

In addition, the platform adopts a single threshold pricing method; that is, the price is set according to the number of drivers $(N)$ available in the system. The pricing strategy has three parameters: low price $p_{L}$, high price $p_{H}$, and threshold $\theta$. When $N \geq \theta$ (here let $\theta=2$ ), the platform pricing $P(N)=p_{L}$; when $N<\theta, P(N)=p_{H}$ [27]. As shown in Figure 2.

From the perspective of the platform, the strategy of income distribution should ensure the maximization of platform profit. The platform profit here refers to the platform's net income, which can be calculated by deducting the drivers' share and the platform costs (operating costs, etc.) from the platform's total income. Then, the platform profit is

$$
T=\left[(1-A) p-T_{C}\right] \lambda(A, p) .
$$

In equation (3), $\lambda(A, p)$ refers to the effective driver arrival rate under the equilibrium state when the extraction coefficient is $\mathrm{A}$ and the price is $p . T_{C}$ refers to the cost of platform.

From the perspective of the government, the operation of online ride-hailing system should ensure the maximization of social welfare. In this paper, social welfare $W_{1}$ is defined as the sum of passenger surplus $(S)$, driver surplus $(D)$, and platform profit $(T)$, as expressed by the following equation:

$$
W_{1}=S+D+T \text {. }
$$

The passenger surplus per ride is the average surplus of passengers who are willing to enter the queuing system. The passenger surplus can be obtained by deducting the price actually paid by passengers from the price that passengers are willing to pay. The transaction volume is the ratio of successful matching in the steady state, which is given by the effective driver arrival rate $\lambda(A, p)$. Then, the overall surplus of passengers can be calculated as follows:

$$
S=\lambda(A, p) \frac{1}{\overline{F_{V}}(p)} \int_{P}^{\infty}(V-p) f_{V}(V) \mathrm{d} V .
$$

Unlike the concept of surplus in traditional economics, the definition of driver surplus is similar to the passenger surplus. The driver surplus per ride can be calculated by deducting the driver's psychological expectation income and the driver's cost (fuel consumption, vehicle damage, etc.) 


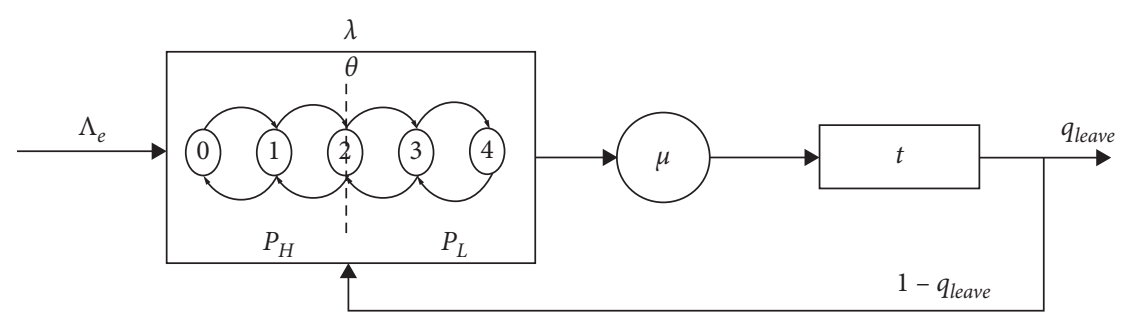

FIgURE 1: Flow process of driver in the system.

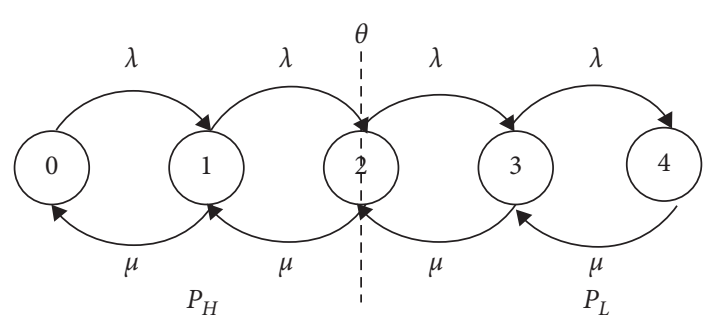

Figure 2: Birth and death process of the drivers in the platform.

from the driver's actual income. Then, the overall driver's surplus can be calculated as follows:

$$
D=\lambda(A, p)\left[\frac{t}{F_{C}(A p / t)} \int_{0}^{(A p / t)}\left(\frac{A p}{t}-C\right) f_{C} \mathrm{~d} C-D_{C}\right] .
$$

3.2. The Dynamic Pricing Model. Dynamic pricing strategies are common in revenue management. In the online ridehailing industry, the service price is usually set by the software. The platform automatically generates a surge multiplier according to time, geographical location, demand, and other factors, to form a dynamic price.

Following the example of Banerjee et al. [13], a threshold-based dynamic pricing method is applied to model the peak time price. In the queuing model constructed above, only the number of vehicles available in the queue is considered to adjust the price, which means that the price is set according to the number of drivers $(N)$ available in the system. The pricing strategy can be expressed as follows:

$$
P(N)= \begin{cases}p_{L}, & N \geq \theta \\ p_{H}, & N<\theta\end{cases}
$$

Among them, $p_{L}, p_{H}$, and $\theta$ represent the low price, high price, and the threshold, respectively. Set $\alpha_{H}=1 / \overline{F_{V}}\left(p_{H}\right)$ and $\alpha_{L}=1 / \overline{F_{V}}\left(p_{L}\right)$, and when the number of drivers is $n$, the platform pricing is $p$, and the driver's income distribution proportion is $A$, the equilibrium driver arrival rate in the online ride-hailing system is defined as $\lambda(A, p, n)$. In order to simplify the influence of the number $(n)$ on the results, we use the equilibrium driver arrival rate under the large market limit $\left(\lambda(A, p)=\lim _{n \rightarrow \infty}(\lambda(A, p, n) / n)\right)$ to study the maximization of social welfare. The arrival rate of drivers meets the following requirements:

$$
\lambda(A, p)= \begin{cases}\mu_{0} \overline{F_{V}}\left(p_{L}\right), & p_{L}>p_{\mathrm{BAL}}, \\ \frac{\lambda_{0}}{q_{\text {leave }}} F_{C}\left(\frac{A p_{H}}{t}\right), & p_{H}<p_{\mathrm{BAL}}, \\ \frac{\lambda_{0}}{q_{\text {leave }}} F_{C}\left(\frac{A P}{t}\right), & p_{L} \leq p_{\mathrm{BAL}} \leq p_{H},\end{cases}
$$

where $p_{\mathrm{BAL}}$ is the equilibrium price when the drivers' supply is equal to the passengers' demand.

$$
\begin{aligned}
\mu_{0} \overline{F_{V}}\left(p_{\mathrm{BAL}}\right) & =\frac{\lambda_{0}}{q_{\text {leave }}} F_{C}\left(\frac{A p_{\mathrm{BAL}}}{t}\right), \\
P & =\frac{p_{L}\left[\alpha_{H}-\mu_{0} / \lambda(A, p)\right]+p_{H}\left[\mu_{0} / \lambda(A, p)-\alpha_{L}\right]}{\alpha_{H}-\alpha_{L}} .
\end{aligned}
$$

From the above model, we can understand the leverage effect of fare adjustment in the supply-demand relationship of online ride-hailing market. The driver's income distribution proportion not only is affected by the driver supply, passenger demand, and platform but also affects the platform supply and demand in turn, to guide the rational allocation of resources according to the rules of supply and demand in economics.

On the basis of dynamic pricing, the social welfare maximization model can be expressed as follows:

$$
\max W_{1}= \begin{cases}\mu_{0} \overline{F_{V}}\left(p_{L}\right) f\left(p_{L}\right), & p_{L}>p_{\mathrm{BAL}}, \\ \frac{\lambda_{0}}{q_{\text {leave }}} F_{C}\left(\frac{A p_{H}}{t}\right) f\left(p_{H}\right), & p_{H}<p_{\mathrm{BAL}}, \\ \frac{\lambda_{0}}{q_{\text {leave }}} F_{C}\left(\frac{A P}{t}\right) f(P), & p_{L} \leq p_{\mathrm{BAL}} \leq p_{H} .\end{cases}
$$

The profit maximization model of online ride-hailing platform based on dynamic pricing can be expressed as follows:

$$
\max W_{2}= \begin{cases}\mu_{0} \overline{F_{V}}\left(p_{L}\right)\left[(1-A) p_{L}-T_{c}\right], & p_{L}>p_{\mathrm{BAL}}, \\ \frac{\lambda_{0}}{q_{\text {leave }}} f_{C}\left(\frac{A p_{H}}{t}\right)\left[(1-A) p_{H}-T_{c}\right], & p_{H}<p_{\mathrm{BAL}}, \\ \frac{\lambda_{0}}{q_{\text {leave }}} f_{C}\left(\frac{A P}{t}\right)[(1-A) p-T], & p_{L} \leq p_{\mathrm{BAL}} \leq p_{H} .\end{cases}
$$


The main parameters and meanings involved in this paper are shown in Table 1.

\section{Numerical Experiments}

4.1. The Influence of City Scale on Driver's Distribution. By comparing the situation of different levels of cities, this section investigates the comprehensive influence of different parameters on the pricing strategy and the distribution strategy of the platform.

4.1.1. Case Setup. We divided the following four scenes to represent different levels of cities, whose basic parameters are as shown in Table 2 .

City $A$ : megacity like Shanghai, Beijing. The population of city $A$ is more than 10 million. It has the highest population density and the most developed transportation system. Correspondingly, it has the largest supply and demand. People's consumption level is higher, and the psychological expected price acceptable to passengers and drivers is the highest.

City $B$ : big city like Suzhou, Chongqing. The permanent population is between 5 million and 10 million, and the population density is around 1400 people per square kilometer.

City $C$ : medium-sized city like Nanchang. Its permanent population ranges from 1 million to 5 million. The population density is around 700 people per square kilometer. The supply and demand of online ridehailing are lower than those of cities $A$ and $B$, people's consumption level is not so high, and the service price acceptable to passengers is lower.

City $D$ : small city. The supply and demand of online ride-hailing are relatively low; people's consumption level and the service price acceptable to passengers are the lowest. The permanent population of such cities is often less than 1 million, with a low population density.

4.1.2. Result Analysis. Models proposed in this paper are applied to calculate and obtain Table 3.

The following conclusions can be drawn from Table 3: (1) In cities $A$ and $B$, which have higher population density and higher levels of supply and demand, whether the pricing is high or low, the price in pursuit of platform benefits maximization is higher than that in pursuit of social welfare maximization. (2) In the outputs of the social welfare maximization model of different cities, the driver's income distribution proportion is between 0.5 and 0.7 , while it is between 0.4 and 0.6 in the outputs of the platform benefit maximization model. In the social welfare maximization model, the driver's interests are more protected. (3) Compared with the social welfare maximization model, the platform profit maximization model not only obtains higher platform pricing but also lowers the driver's income distribution proportion. According to the above models, this is mainly because the platform profit is determined based on the drivers' income and platform pricing. The less the drivers' income distribution is, the higher the platform profit will be. In the contrast, social welfare maximization model considers the balance among online ride-hailing platforms, drivers, and passengers. (4) In addition, it is worth noting that, under the same city parameter conditions, although the quantitative value of social welfare includes platform benefits, the ultimate value obtained from the social welfare maximization model is lower than the platform benefit maximization (expect for city $A$ ).

Therefore, given the actual situation, from the government's point of view, if regulations are not issued to provide guidance for the income distribution proportion of drivers on the online ride-hailing platform, it is very likely that the monopoly price of online ride-hailing platform will be equal to the traditional taxi price, and the interests of drivers will be exploited.

4.2. Relationships between Basic Parameters and Driver's Income Distribution Proportion. In this section, numerical examples with different settings of model parameters are used to analyze the relationship between the driver's income distribution proportion and basic parameters in the two models. The conclusions can provide a reference for the government's decision and policy-making.

4.2.1. Example Setup. Set the initial arrival rate of drivers $\lambda_{0}=2500$ vehicles $/ \mathrm{h}$, the initial arrival rate of passengers $\mu_{0}=5000$ Passengers $/ \mathrm{h}$, the average travel time $t=0.5$ hours, and the probability of drivers leaving the system $q_{\text {leave }}=0.8$. Assuming the psychological expected price of drivers and passengers $f_{c}, f_{V} \sim N(3,1)$. The driver cost is 1.11 yuan $/ \mathrm{km}$, and the platform cost is $0.14 p$. By changing the value basic parameters, including the average travel time, the psychological expected price distribution of passengers and drivers, and the probability of driver leaving the system, the influence of basic parameters on driver's income distribution proportion is analyzed and compared in social welfare maximization model and platform profit maximization model, respectively.

\subsubsection{Relationship between Proportion and Average Travel} Time. As shown in Figure 3, as the value of average travel time increases in the model, the driver's income distribution proportion of the two models increases. From the perspective of economics, this is due to the decrease of supply caused by the increase of ride time. In the case of unchanged demand, the platform price will rise, and accordingly, the driver's bonus should also increase. Additionally, comparing the two broken lines, the income distribution proportion of drivers obtained in the platform profit maximization model is lower than that of the social welfare maximization model, which is consistent with the profit purpose of the platform.

Furthermore, in the platform profit maximization model, the average ride time of passengers significantly affects the driver's income distribution proportion. However, in the welfare maximization model, the driver's income 
TABLE 1: Summary of parameters.

\begin{tabular}{|c|c|}
\hline Parameter & Description \\
\hline$\Lambda_{e}$ & Probability of new drivers arriving at the system \\
\hline$t$ & Average travel time \\
\hline$q_{\text {leave }}$ & Probability of driver leaving the system \\
\hline$\lambda_{0}$ & Initial driver arrival rate \\
\hline$\mu_{0}$ & Initial passenger arrival rate \\
\hline$\lambda$ & Effective driver arrival rate \\
\hline$\mu$ & Effective passenger arrival rate \\
\hline$A$ & Driver's income distribution proportion \\
\hline$p$ & Price of each ride \\
\hline$p_{L}$ & Low platform pricing \\
\hline$p_{H}$ & High platform pricing \\
\hline$\theta$ & Threshold \\
\hline$N$ & Number of available drivers \\
\hline$F_{V}$ & Psychological price distribution of passengers \\
\hline$F_{C}$ & Psychological price distribution of drivers \\
\hline$f_{V}$ & Probability density distribution of passenger psychological price \\
\hline$f_{c}$ & Probability density distribution of driver's psychological price \\
\hline$V$ & The expected price of each passenger \\
\hline$C$ & The expected price of each driver \\
\hline$f$ & Expected free time \\
\hline$h$ & Expected ride income \\
\hline$S$ & Passenger surplus \\
\hline$D$ & Driver surplus \\
\hline$T$ & Platform profit \\
\hline$W_{1}$ & Social welfare \\
\hline$W_{2}$ & Platform profit \\
\hline$D_{C}$ & Driver cost \\
\hline$T_{C}$ & Platform cost \\
\hline$p_{\mathrm{BAL}}$ & Balanced price \\
\hline$d$ & Population density \\
\hline
\end{tabular}

TABLE 2: Basic parameter settings of four cities of different levels.

\begin{tabular}{lcccc}
\hline Parameter & City $A$ & City $B$ & City $C$ & City $D$ \\
\hline$D$ & 3.40 & 1.40 & 0.70 & 0.15 \\
$\lambda_{0}$ & 1500 & 1100 & 1000 & 300 \\
$\mu_{0}$ & 3000 & 2200 & 2000 & 600 \\
$q_{\text {leave }}$ & 0.8 & 0.8 & 0.8 & 0.8 \\
$t$ & 0.75 & 0.5 & 0.5 & 0.33 \\
$f_{V}$ & $N(4,1)$ & $N(3,1)$ & $N(3,1)$ & $N(2,1)$ \\
$f_{C}$ & $N(4,1)$ & $N(4,1)$ & $N(2.5,1)$ & $N(3.5,1)$ \\
$D_{C}$ & 0.11 & 0.11 & 0.11 & 0.11 \\
$T_{C}$ & $0.14 \mathrm{p}$ & $0.14 \mathrm{p}$ & $0.14 \mathrm{p}$ & $0.14 \mathrm{p}$ \\
\hline
\end{tabular}

distribution proportion is less significantly influenced by the average travel time.

It is inspired that, in reality, as the price of online ridehailing services is generally determined by online ridehailing platforms, they prefer longer service time [28], which is contrary to the traffic development of today's intensive cities and green transportation cities. However, in the welfare maximization model, the change of average travel time has no obvious effect on the driver's income distribution proportion.

4.2.3. Relationship between Proportion and Psychological Expected Price of Drivers and Passengers. The distribution of the psychological expected price of passengers and drivers, especially the psychological expected price of drivers, determines the supply of labor force in online ride-hailing market.

In Zha et al. [7] research on the labor supply of online ride-hailing platform, they focused on driver's behavioral decision-making and pointed out that the driver's supply decision-making is affected by the driver's psychological expected price and the average hourly income, which is closely related to driver's income distribution in work. Figure 4 shows the relationship between the income distribution proportion and the psychological expected price of drivers and passengers. It can be seen that, with the increase of the psychological expected price of passengers and drivers, the proportion of drivers' income distribution in the two models shows an increasing trend. This is due to the increase of driver supply caused by the increase of the psychological expected price of drivers and passengers, which leads to the increase of driver supply. Therefore, to achieve better social welfare and increase the income distribution proportion of drivers, it is necessary to raise the psychological expected price of passengers and drivers. For drivers, it is realistic to increase the psychologically expected price. For passengers, it can be realized by some methods: improving the quality of service, such as improve the convenience, safety, and comfort of service, which can increase the consumption intention of passengers and improve the price level acceptable to passengers. In addition, the 
TABLE 3: Model outputs of the four cities.

\begin{tabular}{lcccccccc}
\hline & \multicolumn{2}{c}{ City $A$} & \multicolumn{2}{c}{ City $B$} & \multicolumn{2}{c}{ City $C$} & \multicolumn{2}{c}{ City $D$} \\
& $\begin{array}{c}\text { Welfare } \\
\text { maximization }\end{array}$ & $\begin{array}{c}\text { Profit } \\
\text { maximization }\end{array}$ & $\begin{array}{c}\text { Welfare } \\
\text { maximization }\end{array}$ & $\begin{array}{c}\text { Profit } \\
\text { maximization }\end{array}$ & $\begin{array}{c}\text { Welfare } \\
\text { maximization }\end{array}$ & $\begin{array}{c}\text { Profit } \\
\text { maximization }\end{array}$ & $\begin{array}{c}\text { Welfare } \\
\text { maximization }\end{array}$ & $\begin{array}{c}\text { Profit } \\
\text { maximization }\end{array}$ \\
\hline$A$ & 0.64 & 0.57 & 0.57 & 0.44 & 0.47 & 0.36 & 0.47 & 0.43 \\
$p_{L}$ & 4.39 & 4.76 & 2.00 & 3.36 & 1.55 & 1.18 & 2.54 & 1.18 \\
$p_{H}$ & 4.56 & 4.80 & 3.30 & 3.45 & 2.55 & 2.58 & 3.11 & 2.57 \\
$\lambda(A, p)$ & 635 & 488 & 1129 & 981 & 580 & 561 & 185 & 170 \\
$W_{1} / W_{2}$ & 686 & 672 & 1135 & 1409 & 517 & 721 & 145 & 186 \\
\hline
\end{tabular}

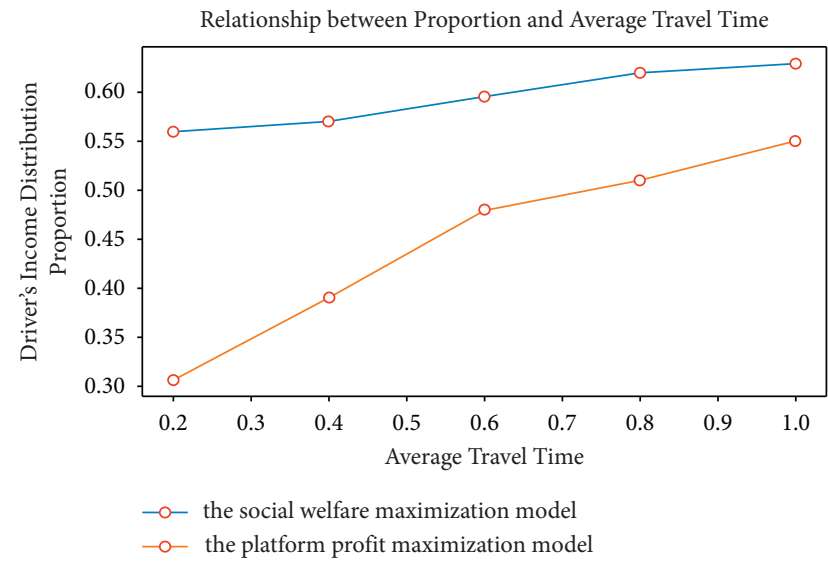

FIGURE 3: Relationship between proportion and average travel time.

increase of the psychological expected price is related to the economic development level of the whole society. When the overall economic level of the society is improved, the acceptable cost of passengers' transportation will be increased, and so will the psychological expected price of drivers and passengers.

4.2.4. Relationship between Proportion and Probability of Driver Leaving the System. The pricing strategy of online ride-hailing platforms and the income distribution proportion of drivers are closely related to the participation of drivers. The probability of drivers leaving the system determines the quantity of supply in the online ride-hailing system. Figure 5 examines the relationship between the income distribution proportion of drivers and the probability of drivers leaving the system. In the social welfare maximization model, with the increasing probability of drivers leaving the system, the driver's income distribution proportion gently increases. This is because, in the dynamic pricing model, the probability of drivers leaving the system hurts the supply of drivers. When the supply of drivers in the system decreases, it will inevitably lead to the increase of platform pricing and income distribution proportion, which is in line with the concept of economics. In the platform profit maximization model, the driver's income distribution proportion also shows an upward trend with the increase of the probability of drivers leaving the system, and the distribution proportion is smaller than that in the social welfare maximization model.

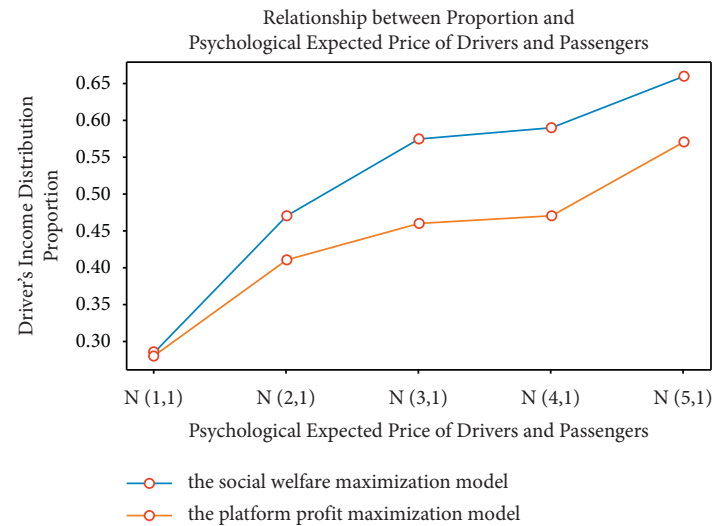

Figure 4: Relationship between proportion and psychological expected price of drivers and passengers.

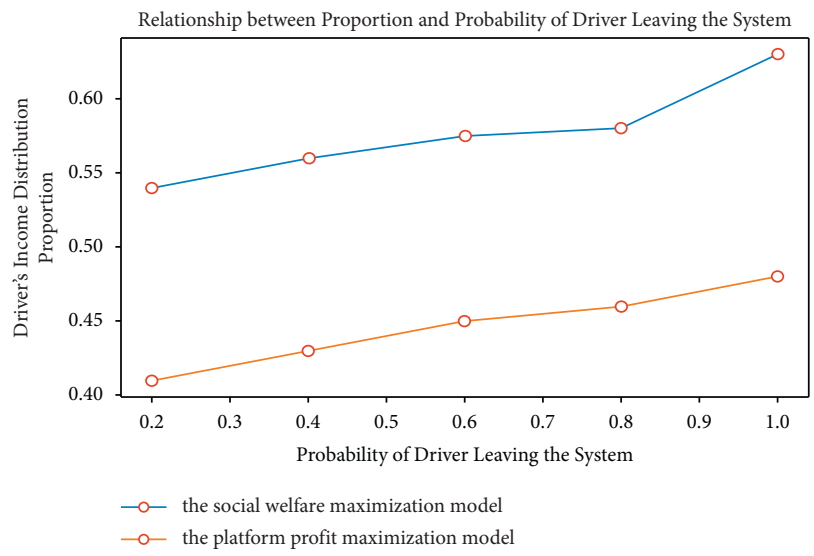

FIGURE 5: Relationship between proportion and probability of driver leaving the system.

4.3. Summary. In this chapter, through the analysis of two examples, we deeply discuss the proportion of the drivers on the online ride-hailing platform.

The first example is based on the basic parameters of supply and demand of four cities of different levels and calculates the extraction coefficient and maximum benefit in the social welfare maximization model and platform benefit maximization model, respectively. It compares the cities to understand the influence of basic parameters of different scales on the extraction coefficient. Finally, we find the following. 
In the former model, the proportion of online ridehailing platform drivers is significantly higher than the latter. This is in line with the reality of the platform's pursuit of interests and also reflects the need for the government to regulate the proportion of drivers.

In the second example, by controlling the variables, we change the average ride time of consumers, the psychological expected price of drivers and passengers, the probability of drivers leaving the system, and other parameters to explore their influence on the driver sharing coefficient in the two models. The example shows the following:

(1) With the increase of any parameter, the sharing coefficient of the two models will rise, but the rising rate is not the same; especially, the psychological expected price of the driver/passenger has a greater impact on the driver's proportion.

(2) At the same time, in the process of each parameter change, the sharing coefficient of the social welfare maximization model is significantly higher than that of the platform benefit maximization model.

\section{Conclusions and Suggestions}

Online ride-hailing platforms have operated in cities worldwide. In response to the lack of transparency and consistency in the pricing and income distribution of online ride-hailing platforms, this study investigated the mechanism of pricing and income distribution.

The contributions of this paper are as follows: (1) Queuing system model and birth and death process theory are introduced to describe the driver's flow process in the network. (2) The social welfare maximization model and the platform benefit maximization model are constructed, from the government's and platform's standpoint, respectively, based on the psychological expected price curve of passengers and drivers. (3) Numerical experiments are performed to analyze the driver's income distribution under the conditions of different settings. The results indicated that the income distribution proportion of drivers is higher in the context of social welfare maximization than that in the context of platform profit maximization. This is consistent with the reality of the platform's pursuit of interests. Moreover, the average riding time of consumers has little influence on the proportion of withdrawal in the context of social welfare maximization, while the psychological expected price of drivers and passengers has a significant impact on it.

Therefore, to maximize the social welfare and protect the driver's rights and interests, the government is suggested to flexibly regulate the pricing and the income distribution of online ride-hailing platforms on the premise of legal compliance. Government can limit the commission charged by the platform and set the lower limit of driver's proportion. First, the monopolized online ride-hailing platform charges a fee for the upper limit of each transaction at most. The setting of the upper limit maximizes the total transaction or the realized demand which is proportional to the consumer surplus. As long as the price is surging, the surge will be transferred to the driver's income to the maximum extent. Additionally, a lower limit should be set to the income distribution proportion of drivers. The setting of a lower limit guarantees the basic income of drivers in the monopolistic and opaque market. Moreover, in the long run, according to the impact of different parameters on the driver's income distribution proportion, the government can encourage long-distance online ride-hailing, develop bike-sharing and tram sharing in short-distance travel, improve residents' income and consumption level, maximize social welfare in urban development, and continuously improve the psychological expected price of consumers and drivers.

This study also has some limitations: for example, this paper only considers the impact of a single factor on the driver's income distribution ratio and does not consider the comprehensive impact of multiple factors. In addition, there is no existing data available online for ride-hailing trips which can be used to test the models. Therefore, these can be considered in future analysis, and other factors, such as spatial differences, traffic congestion, and driving costs, can also participate in discussions.

\section{Data Availability}

The data used to support the findings of this study are included in the article.

\section{Conflicts of Interest}

The authors declare that they have no conflicts of interest.

\section{Acknowledgments}

This work was supported by the National Natural Science Foundation of China (Grant no. 71734004).

\section{References}

[1] Y. Wang, J. Chen, N. Xu, W. Li, Q. Yu, and X. Song, “GPS data in urban online car-hailing: simulation on optimization and prediction in reducing void cruising distance," Mathematical Problems in Engineering, vol. 2020, Article ID 6890601, 2020.

[2] T. Wu, M. Zhang, X. Tian, S. Wang, and G. Hua, "Spatial differentiation and network externality in pricing mechanism of online car hailing platform," International Journal of Production Economics, vol. 219, pp. 275-283, 2020.

[3] Q. Yu, H. Zhang, W. Li et al., "Mobile phone data in urban bicycle-sharing: market-oriented sub-area division and spatial analysis on emission reduction potentials," Journal of Cleaner Production, vol. 254, Article ID 119974, 2020.

[4] S. Xu, X. Yang, and K. Peng, "Research on the proportion of online car-hailing and cruising taxi taking-based on the perspective of the company enterprise distribution mode of online taxi hailing," Price theory and practice, vol. 2019, no. 10, pp. 137-140, 2019, in Chinese.

[5] W. Jiang, H. Zhang, L. Yin et al., "GPS data in urban online ride-hailing: the technical potential analysis of demand prediction model," Journal of Cleaner Production, vol. 279, 2021.

[6] D. N. Anderson, “"Not just a taxi?” For-profit ridesharing, driver strategies, and VMT," Transportation, vol. 41, no. 5, pp. 1099-1117, 2014. 
[7] L. Zha, Y. Yin, and H. Yang, "Economic analysis of ridesourcing markets," Transportation Research Part C: Emerging Technologies, vol. 71, pp. 249-266, 2016.

[8] Q. Yu, W. Li, D. Yang, and H. Zhang, "Mobile phone data in urban commuting: a network community detection-based framework to unveil the spatial structure of commuting demand," Journal of Advanced Transportation, vol. 2020, Article ID 8835981, 15 pages, 2020.

[9] L. Zha, Y. Yin, and Y. Du, "Surge pricing and labor supply in the ride-sourcing market," Transportation Research Part B: Methodological, vol. 117, pp. 708-722, 2018.

[10] N. T. Fellows and D. E. Pitfield, "An economic and operational evaluation of urban car-sharing," Transportation Research Part D: Transport and Environment, vol. 5, no. 1, pp. 1-10, 2000.

[11] Y. Chang, "Economic thinking on the price war of mobile software," Price theory and Practice, vol. 2014, no. 4, pp. 116-118, 2014, in Chinese.

[12] Y. Xia, J. Zhu, and Y. Jiang, "Research on peak pressure relief mechanism of online car Hailing based on dynamic price," Computer and digital engineering, vol. 48, no. 12, pp. 29122918, 2020, in Chinese.

[13] S. Banerjee, C. Riquelme, and R. Johari, "Pricing in ride-share platforms: a queueing-theoretic approach," SSRN Electronic Journal, vol. 2015, Article ID 2568258, 2015.

[14] T. Rangel, J. N. Gonzalez, J. Gomez, F. Romero, and J. M. Vassallo, "Exploring ride-hailing fares: an empirical analysis of the case of Madrid," Transportation, vol. 2021, 2021.

[15] L. U. Ke, J. Zhou, and H. E. Xin, "Research on pricing strategy of ride-hailing platform considering user's preference of service quality," Soft Science, vol. 32, 2018.

[16] Y. Lu, Y. Qi, S. Qi, Y. Li, H. Song, and Y. Liu, "Say No to price discrimination: decentralized and automated incentives for price auditing in ride-hailing services," IEEE Transactions on Mobile Computing, vol. 2020, no. 99, p. 1, 2020.

[17] Z. Xu, Y. Yin, and J. Ye, "On the supply curve of ride-hailing systems," Transportation Research Part B: Methodological, vol. 132, pp. 29-43, 2020.

[18] Z. Liu and Z. Cai, "Political economy analysis of income distribution mode in sharing economy-taking China's internet private car industry as an example," Political economy review, vol. 10, no. 02, pp. 162-177, 2019, in Chinese.

[19] Y. Xie, "Research on bounded rational game behavior of stakeholders in online ride-hailing," Master's thesis, Xiangtan University, Xiangtan, China, in Chinese, 2018..

[20] F. Danlei, "Profit analysis of taxi software platform based on sharing economy," China business theory, vol. 2021, no. 9, pp. 9-11, 2021, in Chinese.

[21] C. Xiang, "Legal regulation of online ride-hailing: logic and thinking -- also on the interim measures for the management of online booking taxi service," Journal of Southwest University of political science and law, vol. 19, no. 6, pp. 53-62, 2017, in Chinese.

[22] L. Fang and X. Zhong, "Research on the development path of online car Hailing Market -- Based on the dual perspective of internal competition and government supervision [J/OL]," Financial and economic treatise, vol. 2021, in Chinese, 2021.

[23] X. Lai, "Transparent pricing rules for online car Hailing should be the basic consensus of the industry," Consumer daily, vol. 2021, in Chinese, 2021.

[24] J. Xia, "A willful online car Hailing platform needs reasonable transparency," China economic times, vol. 2021, in Chinese, 2021.
[25] M. Lin, "Promoting standardized management of online car Hailing," Anhui Daily, vol. 2021, in Chinese, 2021.

[26] Q. Li, "Research on regulatory policy of online ride-hailing in China from the perspective of government regulation," Shandong University, vol. 2017, in Chinese, 2017.

[27] T. Hu and Y. Zhang, "Dynamic pricing strategy of online ridehailing platform," Shandong Science, vol. 33, no. 2, pp. 79-90, 2020, in Chinese.

[28] Y. Li and S. H. Chung, "Ride-sharing under travel time uncertainty: robust optimization and clustering approaches," Computers \& Industrial Engineering, vol. 149, Article ID 106601, 2020. 